PAPER • OPEN ACCESS

Radio-frequency waveform investigation for ion transport within the RFQcb at ISOLDE's Offline 2 facility

To cite this article: S. Warren and T. Giles 2021 JINST 16 P07058

View the article online for updates and enhancements.
You may also like

\begin{tabular}{l} 
- Commissioning of the FRIB RFQ \\
H. Ren, E. Pozdeyev, D. Morris et al. \\
- Deuteron beam commissioning of the \\
linear IFMIF prototype accelerator ion \\
source and low energy beam transport \\
\hline N. Chauvin, T. Akagi, L. Bellan et al. \\
- CW RFQ design and investigation for \\
\hline multi-charge-state acceleration of \\
radioactive beams from BISOL \\
P.P. Gan, H.P. Li, Z. Wang et al.
\end{tabular}

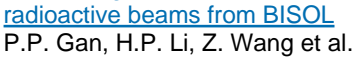




\section{Radio-frequency waveform investigation for ion transport within the RFQcb at ISOLDE's Offline 2 facility}

\section{S. Warren* and T. Giles}

CERN,

Esplanade des Particules 1, 1211 Geneva, Switzerland

E-mail: stuart.warren@cern.ch

Aвstract: A theoretical and practical investigation into the relation of applied waveform, voltage, and frequency of the quadrupole potential applied to the Radio-Frequency Quadrupole cooler and buncher (RFQcb) used at the ISOLDE facility and the Offline 2 facility. Non-radioactive ion beams were transported through the RFQcb over a range of quadrupole parameters to quantify beam transport efficiency via pre and post beam current measurements. Maximum beam transport was directly compared to the theoretical model of ion stability within an oscillatory quadrupolar field via the solutions of the Hill's equation (shown within this work) for each waveform applied the RFQcb and over several masses $20 \mathrm{Ne}, 40 \mathrm{Ar}, 84 \mathrm{Kr}$ and $131 \mathrm{Xe}$. The result of this work has led to interesting findings on the beam transport dependency with the waveform, the potential to reduce the overall costs of future RFQ's, and possible molecular capture break-up options inside RFQcb structure.

Keywords: Beam dynamics; Low-energy ion storage; Instrumentation for radioactive beams (fragmentation devices; fragment and isotope, separators incl. ISOL; isobar separators; ion and atom traps; weak-beam diagnostics; radioactive-beam ion sources); Instrumentation for heavy-ion accelerators

\footnotetext{
${ }^{*}$ Corresponding author.
} 


\section{Contents}

1 Introduction 1

1.1 The RFQcb 2

2 Theoretical approach to stability 2

2.1 Matrix approach to stability 5

$\begin{array}{ll}2.2 \text { Computation } & 7\end{array}$

3 Offline 2-RFQcb testing $\quad 8$

3.1 Test apparatus 8

$\begin{array}{lll}3.2 & \text { 131Xe Waveform tests } & 10\end{array}$

3.2.1 $\mathrm{Kr}$ and Ar testing 11

$\begin{array}{lll}3.3 & \text { Peak performance } & 13\end{array}$

4 Discussion of results $\quad 14$

\section{Introduction}

Beam cooling via a buffer gas has played a significant role in improving the quality of the radioactive ion beams delivered to users at the Isotope mass Separator On-Line facility (ISOLDE) and many others around the world [1-3]. Beams are typically cooled by kinetic energy extraction through stochastic collisions with lesser energetic 'cooler' bodies [4]. Helium is one of most commonly used but there are cases of other impactors, such as, hydrogen [5], and electrons [6]. Buffer gas cooling occurs while the ions are semi-confined within a transverse quadrupolar oscillating field as they traverse over the length of the device. In some cases additional cooling can be attained by trapping the ions within a longitudinal potential well, this increases thermalisation time with the added benefit of accumulating ions to create high instantaneous flux beam bunches. One such device is the ISOLDE Radio-Frequency Quadrupole cooler buncher, RFQcb, which at the ISOLDE facility and named ISCOOL (ISolde-COOLer) [3, 7, 8].

In this study the RF waveform and applied RF potential were investigated for optimum ion transport over a wide range of voltages, frequencies and several atomic masses at the new Offline 2 facility (figure 1) [9]. Offline 2 offers a clone of the existing ISCOOL RFQcb with significantly broader range of RF systems, identical (non radioactive) beam preparations as ISOLDE, and a wide range of beam instrumentation. The majority of the beam measurements were performed on a high dynamic range beam flux secondary electron emission (SEE) detector located at $2 \mathrm{~m}$ downstream of the RFQcb beam extraction [10]. 


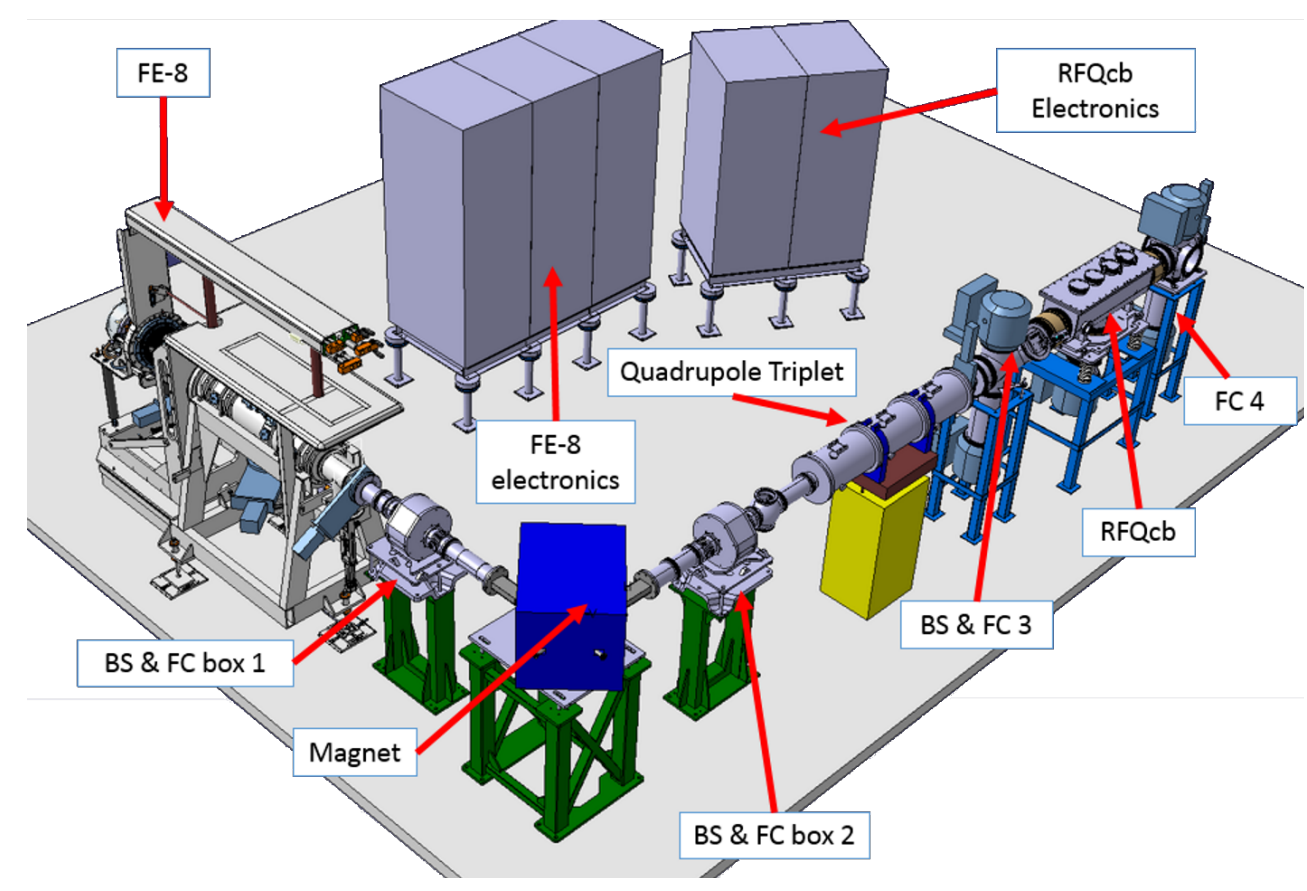

Figure 1. A CATIA rendered false colour image of the Offline 2 separator layout in simplified terms. Where FE-8 is the Front End and ion source, BS \& FC are the Beam Scanners and Farraday Cups, and the magnet provides a mass resolving power of $500 \frac{M}{\Delta M}$. Not shown is the SEE detector but is located $50 \mathrm{~cm}$ downstream of FC 4.

\subsection{The RFQcb}

The ISCOOL RFQcb at ISOLDE and the RFQcb at Offline 2 are structurally identical [9], they are both $80 \mathrm{~cm}$ linear Paul traps [11] filled with low pressure helium $(<0.1 \mathrm{mbar})$ injected $2 / 3$ along the length of the poles towards the injection region. Instead of using segmented poles of the quadrupolar Paul trap to apply a longitudinal DC offsets a series of 25 segmented 'ring electrodes' are installed along the entire length of the quadruple (see figure 2). The DC offset electrodes produces a quasiexponentially decreasing dc offset to guide the cooling ions to the trapping region where they form a potential well of $V_{\text {well }}=0.23 V_{\text {applied }}$ within the quadrupole, where the additional cooling and beam 'bunching' (accumulation) occurs. The injection and extraction assemblies are a series of charged electrodes to decelerate and focus the injected ions into the quadrupole region, and post transport, the extraction assembly re-accelerates the beam to the downstream beam energy of the ion source (see figure 2). These assemblies and their influence on the transport over the RFQcb have been ignored in the mathematical approach to stability and instead focuses on the transport within the trap. However, the matching of these assemblies can significantly alter the transport efficiency over the entire RFQcb and care has been taken to minimise their influence on this study at Offline 2 (see section 3.1).

\section{Theoretical approach to stability}

This approach considers the ions inside the quadrupolar oscillating potential, after the ion injection assembly and before the ion extraction assembly. During transport over the QP region the ions are 


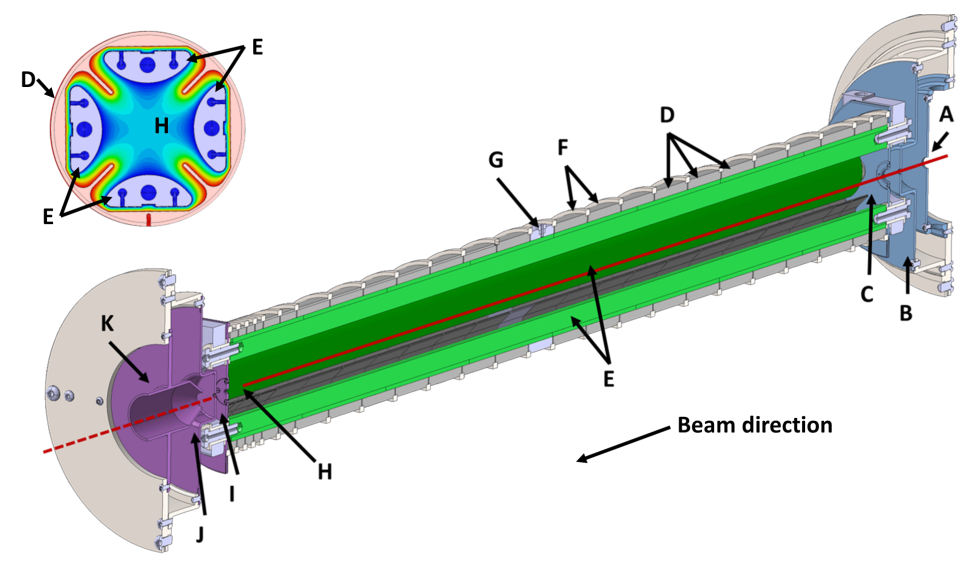

Figure 2. A CATIA rendered false colour image of the core of the ISCOOL RFQcb. Where A),B) and C) are the individually charged injection plates assembly, D) shows the DC axial offset electrodes to apply a potential gradient along the beam axis, E) are the $80 \mathrm{~cm}$ long poles of the quadrupole, F) are ceramic insulators, $\mathrm{G}$ ) is the helium gas injection point (located centrally here), $\mathrm{H}$ ) is the location where the ions can be trapped, I) is the charged trapping plate, J) and K) are the individually charged ion extraction plates. The red line represents the ion beam optical axis where the beam passes in the direction of $\mathrm{A}$ to $\mathrm{H}$ through the buffer gas and the RF field. (Top left) the cross-sectional view of the RFQcb core with false colour image of the effective potential from the axial electrode where $V_{\text {well }}=0.23 V_{\text {applied }}$ at the location $\mathrm{H}$ of potential applied on D.

continuously oscillating between diverging and converging trajectories (w.r.t. to the longitudinal optical axis). The scattering caused by the collisional 'cooling' process is guided back to the optical axis by the QP potential. The proper tuning of these two parameters, scattering from buffer gas, and driven quadrupolar potential to guide ions back, constrains the ions along the longitudinal optical axis throughout the transport. Hence, the stability of the ions within the quadrupole is interlinked with buffer gas and quadrupolar potential. The helium (buffer) gas within the RFQ will create minor perturbations to stability, given the size of the two impactors the mean free path of helium and ion (Xe) can be estimated to be $10-1 \mathrm{~mm}$, indicating semi-infrequent collisions in the intermediate or Knudsen region. The perturbations to the stable ion motion can be modelled via the simple damped resonator equation $\omega=\omega_{0} \sqrt{1-\gamma^{2}}$, where $\omega_{0}$ is the stable frequency without buffer gas, $\omega$ is the perturbed frequency and $\gamma$ is the damping coefficient in the presence of buffer gas. In this work it is assumed that the low pressure $(p<0.1$ mbar) buffer gas will serve as stochastic cooling and the 'damping' caused to the ion motion will be very 'light', where $\gamma \ll 0.1$, and serve to only broaden the stable frequency regions $\omega_{0}$. The influence of this is small but not negligible, it adds a significant level of difficulty to the calculations for stability and will not be included in these computations. This remains valid for stability for small transverse ion amplitudes and velocities but can be seen where it begins to fail.

With the buffer gas influence neglected the common practice to perform a calculation for 'stable' frequencies and potentials required to attain stable periodic ion oscillations during transport via the exact solutions to Hill's equation (equation (2.6)). For the two most common cases the solutions are, the Miessner solutions for switching potential (square), and the Mathieu solutions for sinusoidal potential $[2,5]$. 
The ions amplitude and frequency of oscillation within the RFQcb can been modelled transversely by taking the equations of motion due to the charge in the potential field of a quadrupole,

$$
\begin{aligned}
\phi(x, y, t) & =V_{\mathrm{RF}}(t) \Phi(x, y), \\
\Phi(x, y) & =\frac{x^{2} \hat{x}-y^{2} \hat{y}}{r_{0}^{2}}
\end{aligned}
$$

where $\Phi(x, y)$ is the potential within the quadrupole w.r.t. position $x$ and $y, V_{R F}(t)$ is the zero to peak potential of the applied RF potential, $r_{0}$ is the quadrupole radii, $x, y$ are the deviations w.r.t. the quadrupole central beam axis and $\hat{x}$ and $\hat{y}$ are the respective unit vectors for the horizontal and vertical displacements. Here, it is convenient to substitute $u=x, y, z$ to represent the axis of motion, the force on an ion in direction $u$ is given as $e Z_{i} E_{u}$, where $Z_{i}$ is the charge state of the ion, $e$ is the charge of an electron and $E_{u}$ is the component of the electric field in the direction $u$ given as,

$$
E_{u}=-\frac{d \Phi}{d u}
$$

therefore the ion motion can be given by,

$$
m \frac{d^{2} u}{d t^{2}}=-e Z_{i} V(t) \frac{d \Phi}{d u}
$$

where $m$ is the mass of the ion. For this investigation the $z$-axis is ignored as there is no trapping potentials present, the RFQcb is operating in 'continuous mode' where the net number of ions, $N_{\text {ions }}$, in the RFQcb remained constant over time, $t$, hence $d N_{\text {ions }} / d t=0$.

From the equations of motion we can rewrite them in the common form of the Hill's differential equation given as,

$$
\ddot{u}+\left(\theta_{0}+2 \sum_{n=1}^{\infty} \theta_{n} \cos (2 n t)+\sum_{m=1}^{\infty} \phi_{m} \sin (2 m t)\right) u=0
$$

where $\ddot{u}$ is the acceleration of position $u$ and $\theta_{0}+2 \sum_{n=1}^{\infty} \theta_{n} \operatorname{Cos}(2 n t)+\sum_{m=1}^{\infty} \phi_{m} \sin (2 m t)$ is any arbitrary continuous periodic function. For this derivation the simplified version is given as,

$$
\frac{d^{2} u}{d t^{2}}+f(t) u=0
$$

where $f(t)$ is the periodic influence of the driving QP field. Through implying only periodic solutions, the use of Floquet's theorem $f(t+\pi)=f(t)$, and for the case special case where $m=0$ and $n=0,1$, the Mathieu differential solution is derived as,

$$
\frac{d^{2} u}{d \xi^{2}}+(a-2 q \cos (2 \xi)) u=0
$$

where $\xi=\pi \frac{t}{T}$ is the dimensionless unit of time, where $t$ is the point along the full period of the waveform $T$ [12], $a$ and $q$ are give as,

$$
q=4 \frac{e Z_{i} V}{m r_{0}^{2} \omega^{2}} \quad \text { and } \quad a=\frac{8 e Z_{i} U}{m r_{0}^{2} \omega^{2}}
$$

where $e Z_{i}$ is the charge of the ion and $U$ is the DC offset component of the RFQcb electrodes [13]. 


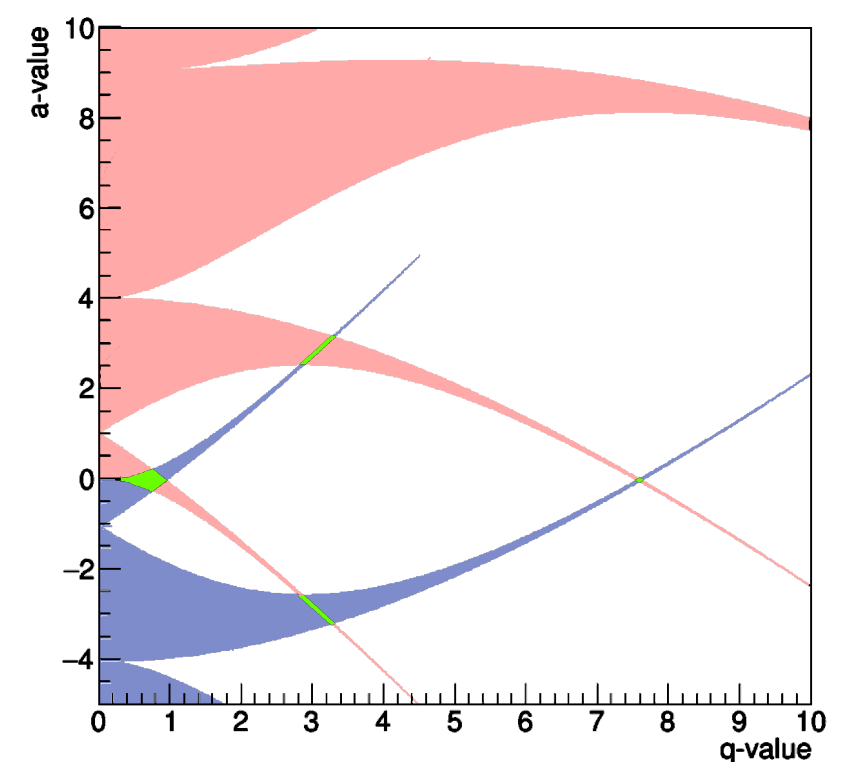

Figure 3. The stable regions for a sine wave function over a range of $a$ and $q$ values. Where the stability in the $\mathrm{x}$-axis (red) is overlaid with the stability in the y-axis to provide stability regions (green) of ion motion within the quadrupolar field.

In many cases ion stability is often given with low values of $q$ and $a$ (see figure 3), where the stability in $y$ is overlapped with the stability in $x$ via $f_{x}(a, q)=f_{y}(-a, q)$. This provides regions in both $x$ and $y$ where stability for ions within the quadrupole reside, for larger values of $a$ and $q$ it is clear from figure 3 and figure 4 that alternative 'regions of stability' exist $(q \approx 1,8,22,46, \ldots)$ and are strongly waveform dependant. This can be seen when we consider the zero DC offset potential, where $a=0$, and the location of stability along the $q$ axis in figure 4 where stability varies significantly depending on the waveform chosen. For example sinusoidal solution to stability overlaps the square waveform initially $(a=0, q<1)$ but as the value of $q$ increases they significant drift away from each other. To obtain these higher $q$ and $a$ values to stability and solutions for any arbitrary applied potential waveform the Hill's equation must be solved.

\subsection{Matrix approach to stability}

The method applied here to describe ion stability is given in detail in [14] where the solutions are based on two fundamental characteristics: linearity and periodicity. Consider a pair of independent solutions $u_{1}(\xi)$ and $u_{2}(\xi)$ to equation (2.6) through one period of the applied RF waveform $0<\xi<T$, where $\xi$ is any point along the period $T$ of the waveform, these solutions can be defined at their initial condition as,

$$
u_{1}(0)=1 ; \quad u_{1}(0)^{\prime}=0 ; \quad u_{2}(0)=0 ; \quad u_{2}(0)^{\prime}=1
$$

where $u_{n}^{\prime}(\xi)=d u_{n} / d \xi$ and $n$ denotes a point later in time. Because equation (2.6) is linear the general solution can be expresses as a superposition of two independent particular solutions,

$$
u(\xi)=x_{0} u_{1}(\xi)+v_{0} u_{2}(\xi)
$$


where $x_{0}=x(t=0)$ and $v_{0}=v(t=0)$, and since the solutions to $u_{1}(\xi)$ and $u_{2}(\xi)$ may be calculated for any time $\xi$, the above is valid for any time along the period $\xi$. However, we need to calculate the solutions through one period of the applied RF waveform. Due to the periodicity of equation (2.6) the same solutions $u_{1}(\tau)$ and $u_{2}(\tau)$ will be valid for $\tau=\xi-T$ during the second period of $T<\xi<2 T$ and the same for $\tau=\xi-n T$. From equation (2.10) we can express $u(\xi)$ as,

$$
u(\xi)=x_{n-1} u_{1}(\tau)+v_{n-1} u_{2}(\tau)
$$

It is now possible to calculate (via equation (2.11)) the trajectory of an ion at any given time, $\tau$, along the applied RF waveform. It is more convenient to rewrite equation (2.11) in a form more familiar to beam optics calculations,

$$
\left[\begin{array}{l}
x_{n+1} \\
v_{n+1}
\end{array}\right]=M \cdot\left[\begin{array}{l}
x_{n} \\
v_{n}
\end{array}\right]=M^{n} \cdot\left[\begin{array}{l}
x_{0} \\
v_{0}
\end{array}\right]
$$

where

$$
\left[\begin{array}{ll}
u_{1}(T) & u_{2}(T) \\
v_{1}(T) & v_{2}(T)
\end{array}\right]=\left[\begin{array}{ll}
m_{11} & m_{12} \\
m_{21} & m_{22}
\end{array}\right]=M
$$

Methods to calculate the n-th power of a 2x2 matrix are given in [14] and $M$ is from this point referred to as the transport matrix.

The calculation of the general solution to the Hill's equation is now reduced to the calculation of two independent solutions through one period.

There are special cases where the solutions can be analytically computed, such as the previously mentioned Meissner and Mathieu solutions. For the non-analytical solutions to the transfer matrix, $M$, each moment of $\tau$ inside the first period of $T$ must be calculated. By applying the constraints given by beam optics stability, where the matrix $M$ has special properties due to Liouville theorem, the determinate of the matrix must be conservative, $\operatorname{det}(M)=1$, and such that each momentary instance of time, $\tau$, is also conservative $\operatorname{det}(M(\tau))=1$. Therefore, the time varying voltage of the waveform $\left(V_{R F}(\tau)\right)$ over the period $\tau=\frac{t}{T} \pi-n T=\xi-n T$ can be distributed in to series of $n$ fixed discreet values of $\tau_{i}$,

$$
\sum_{i=0}^{n} V\left(\tau_{i}\right)=\int_{0}^{\xi} V(\xi) d \xi \quad \text { where } \quad n=\xi / \Delta \xi
$$

such that $\Delta \xi$ is sufficient to invoke the assumption,

$$
\left.\mid V\left(\tau_{i+1}\right)-V\left(\tau_{i}\right)\right)|-| V\left(\Delta \xi_{i}\right) \mid \approx 0
$$

where at any discrete waveform point $V\left(\tau_{i}\right)=V(\xi)$ and $\frac{d V(\xi)}{d \xi} \approx 0$. This allows for the possibility to solve equation (2.6) for each instance of $\tau_{i}$ as the voltage $V(\xi)$ is constant over the discrete instance, $\Delta_{i}$ where $\Delta_{i}=\tau_{i}-\tau_{i+1}$. The ion position and velocity at the beginning of the interval $i$ are $x_{i}$ and $v_{i}$ are given as,

$$
\left[\begin{array}{l}
x_{i+1} \\
v_{i+1}
\end{array}\right]=M_{i}\left(f_{i}, \Delta_{i}\right) \times\left[\begin{array}{l}
x_{i} \\
v_{i}
\end{array}\right]
$$

where,

$$
M_{i}\left(f_{i}, \Delta_{i}\right) \times\left[\begin{array}{cc}
\cos \left(\Delta \sqrt{f_{i}}\right) & \frac{1}{\sqrt{f_{i}}} \sin \left(\Delta \sqrt{f_{i}}\right) \\
-\sqrt{f_{i}} \sin \left(\Delta \sqrt{f_{i}}\right) & \cos \left(\Delta \sqrt{f_{i}}\right)
\end{array}\right]
$$


where $f_{i}$ is the discreet momentary value of $f(\xi)$ given by $f(\xi)=a-2 q(\xi)$, where $a$ and $q$ are described by equation (2.8) and the instance of $\tau_{i}$ with width $\Delta$. For the case of $f<0$ the hyperbolic trigonometric functions are substituted (e.g. $\cos \left(\Delta \sqrt{f_{i}}\right)$ to transform to $\cosh \left(\Delta \sqrt{-f_{i}}\right)$ ) and with equation (2.12) we have the transport matrix $M=M_{n} \times M_{n-1} \times \ldots M_{1}$ after $n$ segments of $f\left(\tau_{i}\right)$ over a full period of $\xi$ corresponding to the applied waveform $V(T)$. From previously, the stability is given by Liouville theorem as the trace of the matrix $M,|\operatorname{Tr}(M)|=\left|m_{11}+m_{22}\right|=\beta$ where if $\beta>2$ the ion's motion will be increasing and unstable over the period of $\xi$ and if $\beta<2$ the motion is stable $[12,15]$. This gives us the parameter for stable transport as

$$
\frac{|\operatorname{Tr}(M)|}{2} \leq 1
$$

From the nature of equation (2.6) and equation (2.18) we can invoke that stability in both $\mathrm{x}$-axis and $\mathrm{y}$-axis will correspond to permitted transport across the RFQ. Hence, a solution where $\left|\operatorname{Tr}\left(M_{x}\right)\right| / 2 \leq 1$ and $\left|\operatorname{Tr}\left(M_{y}\right)\right| / 2 \leq 1$ is stable through the entire RFQ transport. As and example the stable (where $\beta<1$ ) solutions for a small range of $a$ and $q$ values in the x-axis has been computed for three waveforms and been given in figure 4 . To ensure the code was correctly behaving correctly the Mathieu solutions were compared to the computed sinusoidal solutions and found to match. Additionally, the code was checked over the values of $a$ and $q$ given in [15] for the ranges provided and found to match for all given waveforms.

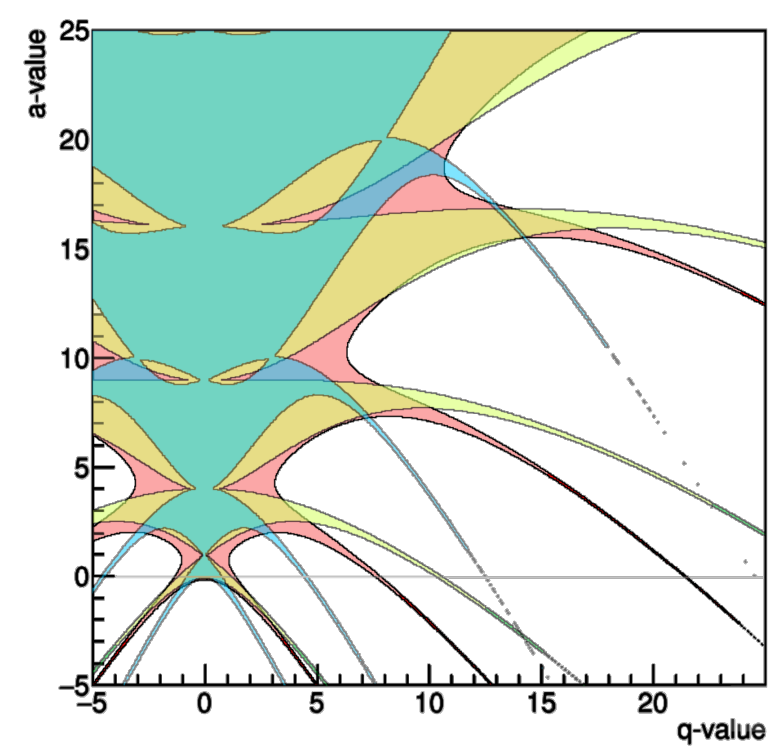

Figure 4. The stability solutions for the Hill equation for a range of $a$ and $q$ values (given in eq. (2.8)) calculated within this work for several waveforms for one axis of motion $(x)$. Blue (top layer) is the stability for a square wave, yellow (median layer) is for a triangle wave and red is for sine wave. The stability (as defined in section 2.1) is shown as coloured regions for each waveform, outside these the ion oscillations become chaotic and with ever increasing amplitude.

\subsection{Computation}

All computations for stability were performed using a single NVIDIA TITAN V GPU with $\mathrm{C}++/$ CUDA programming language for the solutions to the matrices described in section 2.1. The 


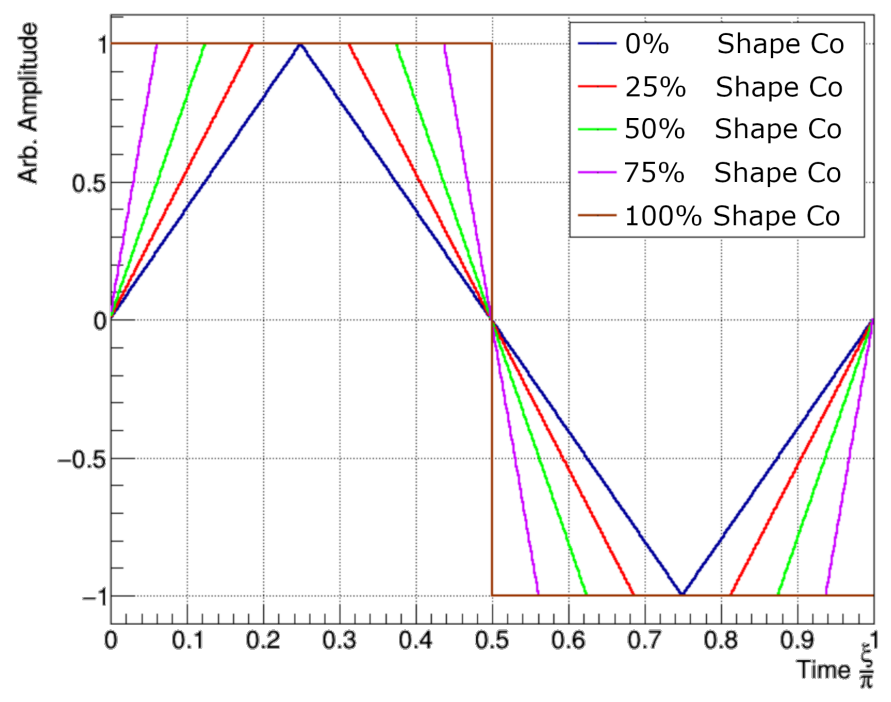

Figure 5. The applied waveforms with their respect shape co-efficient values over the time period $\frac{\xi}{\pi}$. The shape co-efficient is defined as the percentile of a the period at the maximum of minimum value, such that $50 \%$ represents half of the period at the maximum/minimum potential and the remaining $50 \%$ transitioning between.

solutions were computed with 1000 samples of $\tau_{i}$ per waveform over $T$ and fidelity of $\Delta q=10^{-9}$ for systems with $a=0$ over a range of $q=0$ to 250 . For full a vs q maps $\Delta a=\Delta q=10^{-6}$ from $a=q=-50$ to 50 giving a very large number of computations per map. Although a relatively simple calculation the number of operations becomes very large, the matrix approach does offer significant improvements in mathematical simplicity but it also introduces a large amount of operations per data point. Fortunately, the advances in parallel computing GPU hardware and software means this problem can be distributed over the thousands of threads available on each GPU processor such that each waveform calculation took approximately 3 hours to compute with double pointer precision. This value can be significantly improved by intelligent choice of number precision and memory management but already is a very significant speed up over the small number of threads available on a conventional CPU C++ code/method.

\section{Offline 2 - RFQcb testing}

For the purpose of this investigation we wish to directly compare the mathematical model of stability (given in section 2.1) to the ISOLDE RFQcb transport over a wide range of q-values and for a selection of waveforms. The waveforms selected for the investigation were, sinusoidal, triangle and a trapezoid with varied flat-top duties (shown in figure 5).

\subsection{Test apparatus}

The investigation was carried out at the Offline 2 test facility, as previously described section 1.1 and shown in figure 1 . The ion source was a VD5 plasma ion source [16] on a standard ISOLDE target base. The ion source was supplied with gas mixture of five even parts $\mathrm{He}, \mathrm{Ne}, \mathrm{Ar}, \mathrm{Kr}$ and $\mathrm{Xe}$ (at their own naturally occurring isotopic abundances) and regulated to 1250 mbar absolute pressure 
for the duration of the experiment. The gas was forced through a calibrated leak of $10^{-6} \mathrm{mbar}_{1} \mathrm{~s}^{-1}$ into the hot ion source via a short Ta $1 \mathrm{~mm}$ capillary. The beam was matched and transported over the 90 Degree separator magnet to a focal point post separation for maximum resolving power of $495 \frac{m}{\Delta m}$ and matched to the RFQcb optimal acceptance. The Offline 2 RFQcb is mechanically identical to ISCOOL but offers significant improvements: the Pumping capacity pre-injection and post-extraction are double the online version ( 2600 to $6850 \mathrm{l} / \mathrm{s}$ ), the buffer gas injection system is automated and regulated, and the RF system is a continuous Arbitrary Waveform Generator (AWG) with a flat response bandwidth of 0.01 to $20 \mathrm{MHz}$, it can be pushed to $100 \mathrm{MHz}$ if non linearity is acceptable. The gain of the RF system is as follows: $-6 \mathrm{~dB}$ on the LabView PIXe-5413 AWG signal output, $+34 \mathrm{~dB}$ on the primary amplifier [17] (4 Watt BE-RF-IS 4222), $+26 \mathrm{~dB}$ on the secondary amplifier [18] (800 Watt BE-RF-IS 4241). This is then connected over a $50 \Omega$ impedance matched $5 \mathrm{~m}$ high power coaxial cable to an impedance matching transformer to compensate the capacitance of the RFQ poles and give an additional gain of $+10 \mathrm{~dB}$. The reflections form the RFQ poles are isolated by the transformer and attenuated separately via two $2 \mathrm{KW}$ Tenuline 8389-300 load alternators, this gives the total gain of the RF system of $+64 \mathrm{~dB}$.

To ensure continuity between tests, all the systems excluded from the test variables were prepared using the following procedures:

- Ion source - The ion source was heated through restive heating at $330 \mathrm{~A}$ over the line, 5.5 A applied on the solenoid magnet on the target and a potential of $-120 \mathrm{~V}$ over the anode. The system was then left for 24 hours to stabilise and de-contaminate (isotopic contamination's from manufacturing and exposure to atmosphere).

- Helium buffer gas - The helium buffer gas used for the RFQ was injected into the core using a needle valve coupled to a Bronkhorst pressure regulating valve and was set to $1.25 \pm 0.01$ mbar. The helium pressure in the core will be fractional to this value (estimated through Molflow++ [19] to be 0.05-0.1 mbar, but remains constant throughout the experiment duration and optimised at the start to the beam energy).

- Beam matching parameters - The beam optical 'tune' pre and post the separator sector remained constant throughout the experiment. It was optimised with $40 \mathrm{keV} 131 \mathrm{Xe}$ beam with an emittance of $30 \pi \mathrm{mm}$ mrad. Any changes made were purely to steer the beam to optimise mass selection via the mass separator magnet.

- RFQcb RF - A sinusoidal waveform known to operate at ISCOOL was used to confirm functionality of the all the prerequisite systems then optimised before the experiment commenced.

- RFQcb Electrodes - The axial offset electrodes and RFQcb trapping plate were grounded to the platform potential to remove any possible $a$ component occurring throughout the tests. The injection and extraction plate assemblies were optimised with the sinusoidal tune after each mass change and remained constant throughout the test mass.

Post setup, the beam transport efficiency $\left(I_{\text {post }} / I_{\text {pre }}\right)$ over the RFQcb of was measured with large acceptance Faraday cups, FC, up stream and down stream of the RFQcb to confirm 'nominal' operating conditions. Directly comparing the measured beam current on the identical detectors gave 
typical transport efficiencies of $70 \%$ to $80 \%$ were attained but is dependant on the mass (131Xe was $76 \%$ ). This and a repetition of these set up steps were performed at the end of each test to confirm beam and ion source stability throughout.

\subsection{Xe Waveform tests}

A preliminary test beam of $131 \mathrm{Xe}$ was transported while alternating between waveforms to investigate the influence on transport efficiency with the frequency shift of peak transport. The instrument used to read out the post RFQcb transport beam current was the new SEE detector [10] instead of the FC. This provided a significant improvement in read out sensitivity and integration with control systems but cannot absolutely determine the beam current while operating in high flux FC mode. Figure 6 shows the beam intensity measured post transport over three waveforms (sine, triangle and square wave) as the frequency of each waveform was swept from 0.01 to $1.0 \mathrm{MHz}$ in steps of $250 \mathrm{~Hz}$.

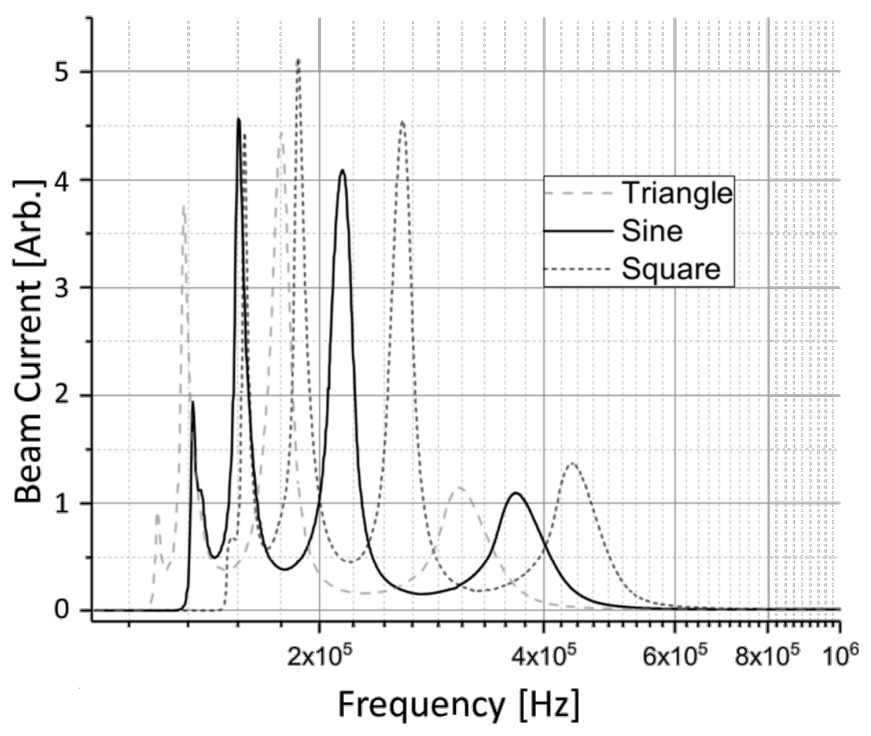

Figure 6. Beam current measured post RFQcb transmission with three waveforms applied at varied frequencies 0.1 to $1 \mathrm{MHz}$. The spectra shows the locations of maximum transmittance and subsequently lesser peaks of transmission for each of the waveforms with constant amplitude of $1 V_{0} p$ applied on the AWG generator. The Beam current from the SEE detector is a relative measurement as it is measuring the secondary beam current generated by the electrons, this makes it more sensitive but not absolute.

The transported beam current with frequency (figure 6) shows several peaks of 'good' transport over the frequency domain. The multiple peaks of 'good' transport can be correlated to the arms of stability given in figure 4 . The optimum transport varies significantly in location with respect to the applied waveform and also shows optimum transport for the square wave. The beam transport improved significantly with increasing values of $q\left(\propto 1 / \omega^{2}\right)$ with significant shifts in the most efficient peak frequency.

The preliminary result prompted an investigation into the parameter space of $q(V, \omega, m)$ given in equation (2.8), in particular, the shape and potential of the applied voltage $V$ and the frequency 
$\omega$. A waveform with a given shape co-efficient (see figure 5) and amplitude, $V$, was used to transport the beam over the RFQcb while varying the frequency (as previously done). The resulting transport spectra (similar to figure 6) becomes increasingly overcrowded with several peaks per spectra overlapping for each shape co-efficient value. To make the data legible and understandable the location of each peak (a region where significant transport was attained) was quantified by taking a minimum beam current threshold of $0.5 \mathrm{nA}$ and taking the peak centre with respect to the full width half maximum to provide the stable frequency of stability $\omega$. This data was then processed via equation (2.8) using the known values for $V, m$ and the measured values of $\omega$ to give to get the corresponding $q$-values. These values were then overlaid with the computed stable solutions for the waveform to provide correlation between the theory and the predicated values, figure 7 .

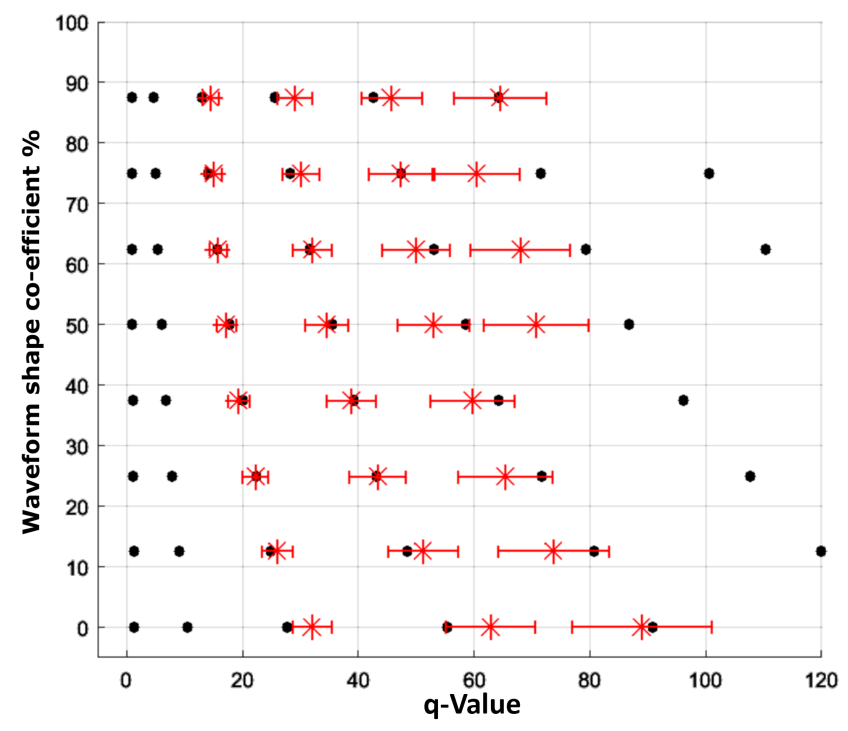

Figure 7. Computed q-values (represented by black circles) plotted against the values calculated q-values from the 131Xe data taken on the RFQcb (represented by the red asterisk) for constant applied voltage in on the AWG. Only the peaks with significant transport are shown ( $>0.5 \mathrm{nA}$ for a beam of $5 \mathrm{nA}$ ) the lower values of $q$ were cut off by this threshold. The calculation and error bars are discussed within the body of the text.

The errors associated with the computed vales (in figure 7) are strongly dominated by the uncontrolled variance in $r_{0}=20 \pm 3 \mathrm{~mm}$ (measured in centre), the quadrupole radii. The mechanical structure of the poles, a $80 \mathrm{~cm}$ long split cylinder rod, sags in the middle of its length and is only supported by the ends mechanically held in place by M8 bolts and two $5 \mathrm{~mm}$ peek pins that deform. The centre of the rod is compressed slightly by the weight of the axial electrodes and relies on the rigidity of the pole to remain in place.

To confirm the relation between computed and calculated values the process was repeated with a range of $V$ and overlaid with the previous results to show the q-values are correlated within error(see figure 8). A similar beam threshold of $0.5 \mathrm{nA}$ was applied to this data and it can be seen as the transmission becomes gradually worse for the lower voltages.

\subsubsection{Kr and Ar testing}

Identical testing was performed (as described in 3.2) with the same set up procedures described in 3.1 but with two alternate masses. This serves as a validation and confirmation of the computed 


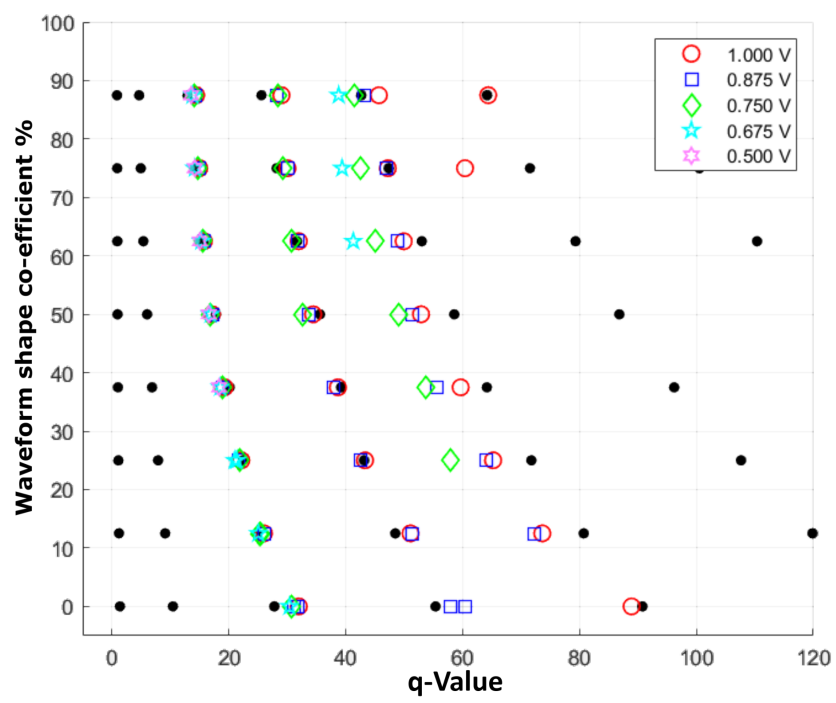

Figure 8. Computed q-values (represented by black circles) plotted against the values calculated q-values for the 131Xe data taken on the RFQcb for a range of $V$ on the AWG. Only the peaks with significant transport are shown $(>0.5 \mathrm{nA}$ for a beam of $5 \mathrm{nA}$ ) the lower values of $q$ were cut off by this threshold. For illustrative purposes and to show correlation the error bars have been removed from the graph.

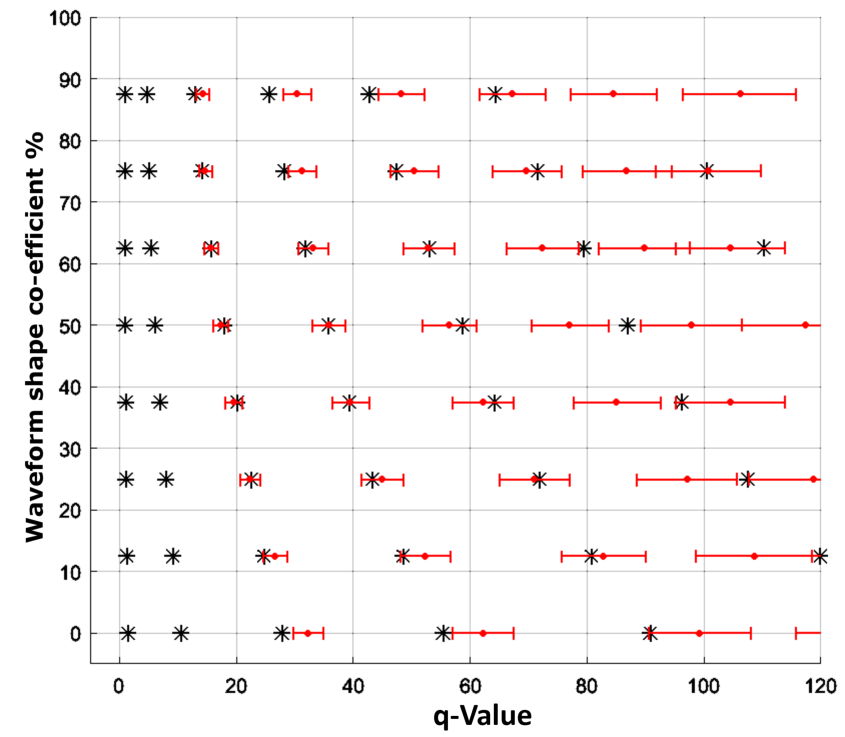

Figure 9. Computed q-values (represented by black asterisk) plotted against the values calculated q-values (represented by the red circle) from the $84 \mathrm{Kr}$ data taken on the RFQcb.

values as the frequency of stable transport relates too the inverse square-root of the mass. The $84 \mathrm{Kr}$ data (figure 9) and the 40Ar data (figure 10) both show a strong correlations with predicted and measured $q$-values over the range of waveforms. 


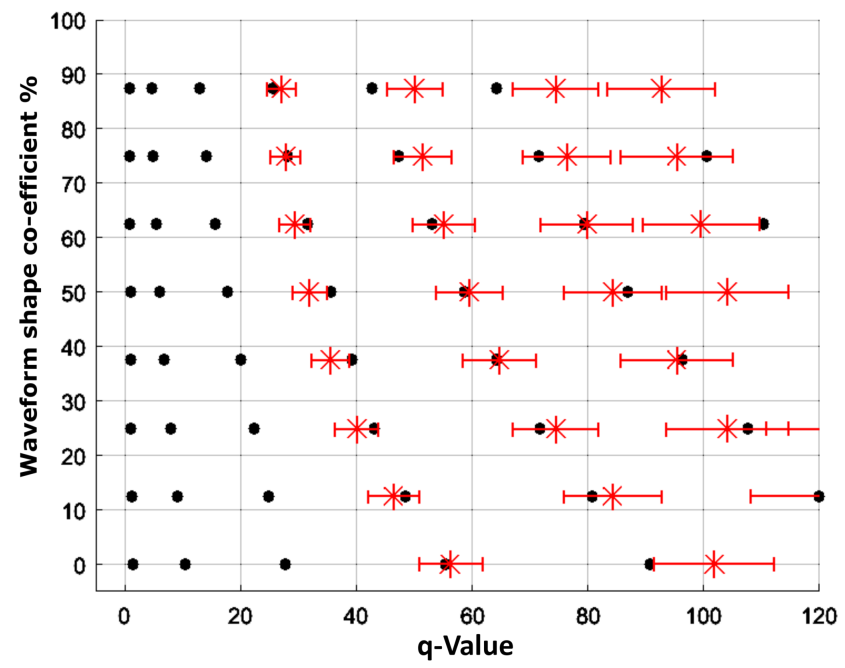

Figure 10. Computed q-values (represented by black circles) plotted against the values calculated q-values (represented by the red asterisk) from the 40Ar data taken on the RFQcb.

\subsection{Peak performance}

Due to the nature of the ion source it was not appropriate to assume the beam properties (especially the ion beam current) remained constant over the long data taking (typically 36 hours per mass) over the previous study section 3.2 data collection. Due to these uncertainties in the beam the maximum transport across the RFQcb could not be fairly determined. Instead, an alternative method was used to determine maximum transport for several masses with different waveforms.

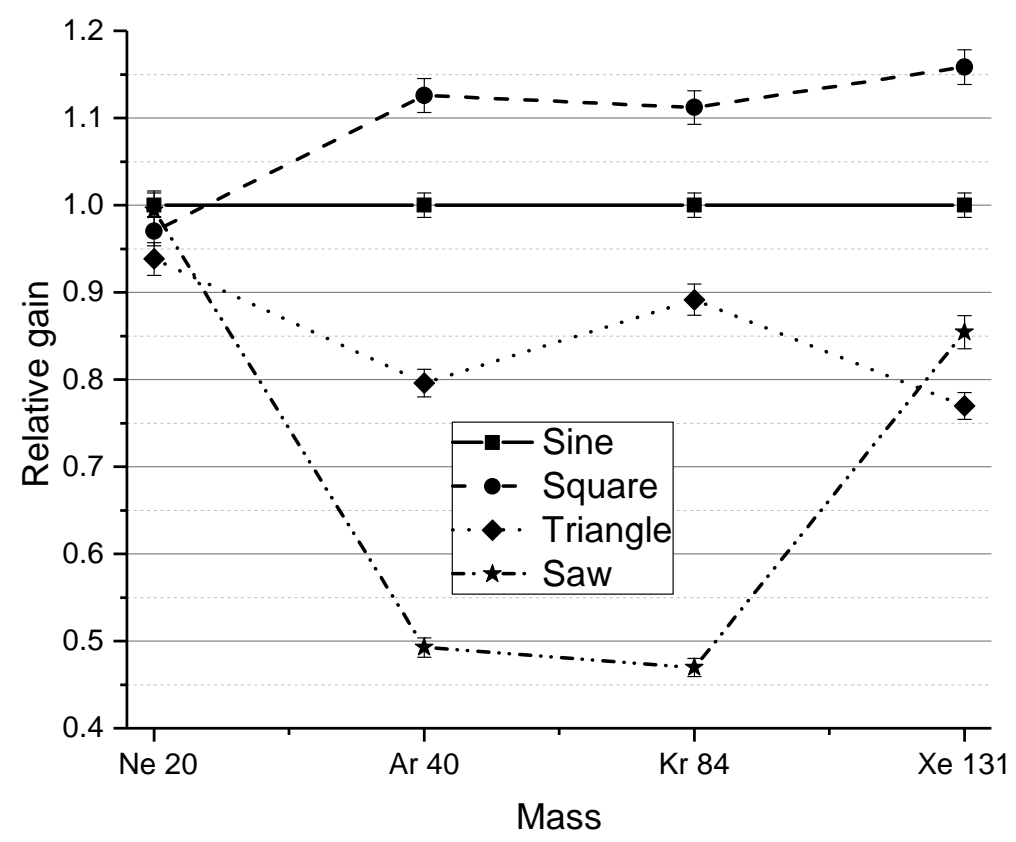

Figure 11. The associated gain and loss in beam transport relative to sine wave RF for several masses over the RFQcb 
The beam was changed quickly changed between masses and the maximum transport was optimised using the standard sine waveform then immediately compared to the maximal transport of alternative waveforms through manual optimisation of frequency. To speed up the process the extremes of the waveform, sine, square, triangle and saw-tooth were used. The waveform transport efficiency was directly probed through FC measurements pre and post the RFQcb transport to provide relative transport efficiency (instead of the SEE detector used for the previous studies) with respect to the standard sine waveform (see figure 11). This gave maximal transport for the square waveform with an increase of $15 \%$ over the traditional sine wave. The transport efficiency was significantly reduced ( -10 to $-25 \%)$ for the triangle waveform over masses greater than $40 \mathrm{Ar}$ and remained significantly worse over the range of masses tested. All the waveforms provided similar (or within error) transport efficiencies for the light mass, $20 \mathrm{Ne}$, indicating an alternative dominating affect influencing transport.

\section{Discussion of results}

The mathematical model and solutions given in section 2 and section 2.1 adhere closely to measured values for the low q values (figure 7 and figure 9), where we would expect the higher frequencies of RF to cause smaller ion oscillation amplitudes. In the region of higher values of $q$ the computed and calculated values begin to deviate, this can be explained by considering the path length (and amplitude) of the ion oscillations, $u$, during one period of the applied waveform, $f(t)$. For the simple case we only consider the time dependant field $E(t) \propto V_{R F}(t)$, the maximum velocity attained will be proportional to the integral of $\frac{d u}{d t} \propto \int_{0}^{\pi} q Z E(t) d t$. Where an aggressive waveform, such as, the $100 \%$ shape co-efficient from figure 5 will experience the maximal field for a longer duration and result in greater acceleration and velocities the transverse plane. The additional path length $d u$ traversed through the helium buffer gas will result in more collisional cooling, leading to thermalisation of the ion beam in a shorter duration. The limit to this is determined by: ion capture (beam injection), where the sudden deceleration into the RFQcb causes the non-normalised emittance, $\varepsilon$, to grow proportionally to the square of the fractional difference in kinetic energies

$\left(\varepsilon_{R F Q c b} \propto \varepsilon \sqrt{\frac{E_{\text {Beam }}}{E_{R E F C b}}}\right.$ without buffer gas cooling), and the maximum permitted deviation from the central beam axis due to beam collisions with the quadrupole structure. To further this hypothesis, beam transport for light mass $20 \mathrm{Ne}$ does not appear to be heavily influenced by the change in waveform (figure 6). 20Ne-4He collisions occurring will result in significant deviations from the optical axis with significant changes in the ion momentum vector; this results in a heavily damped system than that of the heavier ion system. These postulates could be simulated using transport codes with ion-gas collisions for selected frequencies and would be of great benefit to future studies but care would need to be taken to ensure the computations do not become to massive.

These considerations lead to an interesting conclusion for RFQ coolers and the RFQcb (ISCOOL), beam transportation can be attained using many other waveforms and much more importantly alternative waveforms can even give significant gains on the transport over the conventional sine wave. The square wave holds major significance, it can efficiently transport beam over the RFQ with improved transmittance, it is speculated to improve cooling and is significantly cheaper to produce. The amplifiers/waveform generators required for these tests come at a considerable expense and the results here show it is possible to replace these expensive generators (or resonant 
circuits used in many other RFQ coolers around the globe) with a simple set of on/off transistors and DC power supplies. The lack of dependence on resonant systems makes swapping from high to low masses very simple and easy, a very attractive feature for many RFQ's with wide mass ranges.

If we build upon this work and deliberately use the higher $q$ values for transport it opens up the door to the possibility of having several masses contained in the RFQcb via a superposition of several waveforms. An obvious and interesting exploit of this could be the injection of molecular beams of mass $m_{1}$ and the disassociation of the bond within the trap to give the daughter masses $m_{2}$ and $m_{3}$ that are also trapped within the RFQcb. By utilising the solutions given in section 2.1 and careful alteration of the waveform function $f(\xi)$ it becomes possible to manipulate and control the masses held and chaotically rejected the undesired from the trap, see figure 12 .
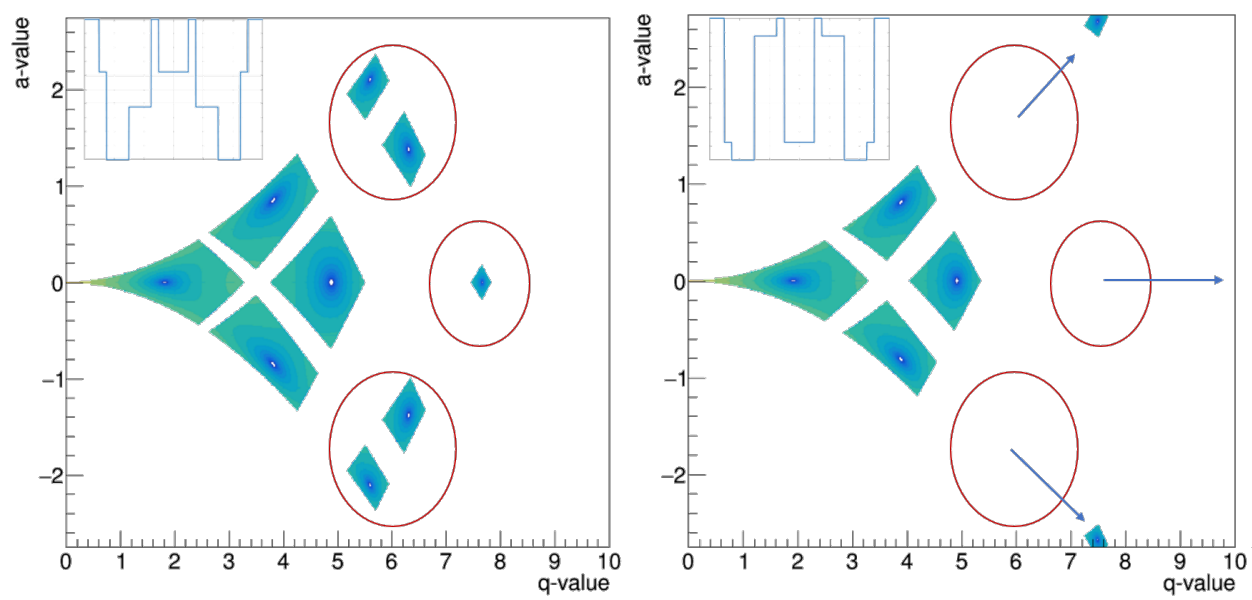

Figure 12. A supposition of two waveforms (shown top left of each figure) with integer periodicity such that by changing the amplitude ratio between one waveform (left) and the next (right) it is possible to manipulate the regions (circled red) of stability and force trapped masses into a chaotic non-trapping region of $q$ and $a$ while also trapping the desired mass.

The trapping component through the use of the DC axial electrodes would require the addition of the $z$-axis to be included into the stability calculations. This will reduce the parameter space for stable transport as it is overlaid with $x$-axis and $y$-axis stability but could also be used to further manipulate additional ion rejection. Future studies should include this influence and the pseudo-potential well for trapping to improve ion delivery to the ISOLDE experiments.

Given all theses benefits future RFQ's designers should strongly consider the square wave driven system with $q$-values higher than the traditionally used to improve the overall transport and the cooling efficiency where capture permits.

\section{Acknowledgments}

The majority of this work would not have been possible to complete without the extensive help of the various groups of CERN. The CERN BE-RF groups technical expertise in RF made it possible to reach such a wide range of frequencies and power never seen before by ISCOOL, the CERN EN-STI-RBS group for allowing the use of the extremely unique Offline 2 facility, the ion source 
engineers for assisting and providing a VD5 ion source for the duration of the experiments. The simulations and the computing power required to simulate the beam transport was only feasible due to the raw computing power of the TITAN V GPU kindly donated by NVIDIA, previous attempts at this with CPU systems were time consuming to the point of impossible to achieve realistically and it is thanks to their donation the majority of the computations were possible.

\section{References}

[1] R. Boussaid, S. Kondrashev and Y.-H. Park, Rare isotope sciences project, in the proceedings of the $5^{\text {th }}$ International Beam Instrumentation Conference (IBIC2016), September 11-15, Barcelona, Spain (2016).

[2] M. Smith, L. Blomeley and P. Delheij, First tests of the TITAN digital RFQ beam cooler and buncher, Hyperfine Inter. 173 (2006) 171.

[3] A. Jokinen, M. Lindroos, E. Molin and M. Petersson, $R F Q$-cooler for low-energy radioactive ions at Isolde, Nucl. Instrum. Meth. B 204 (2003) 86.

[4] A. Kellerbauer, T. Kim, R. Moore and P. Varfalvy, Buffer gas cooling of ion beams Nucl. Instrum. Meth. A 469 (2001) 276.

[5] M. Lewitowicz, The spiral 2 project, Nucl. Phys. A 805 (2008) 519c.

[6] G. Tranquille, Specification of a new electron cooler for the low energy ion ring, leir, Nucl. Instrum. Meth. A 532 (2004) 399.

[7] E. Mané et al., An ion cooler-buncher for high-sensitivity collinear laser spectroscopy at ISOLDE, Eur. Phys. J. A 42 (2009) 503.

[8] I. P. Aliseda, New develepmonts on preporation of cooled and bunched beams at ISOL-facilaties: The ISCOOL project and rotating wall cooling, $\mathrm{PhD}$ thesis, University Politecenica de Catalunya, Spain (2006).

[9] S. Warren, T. Giles, C.M. Pequeno and A. Ringvall-Moberg, Offline 2, Isolde's target, laser and beams development facility, Nucl. Instrum. Meth. B 463 (2020) 115.

[10] S. Warren, T. Giles, C.M. Pequeno and A. Ringvall-Moberg, A new fast time of flight detector for single ion counting to high flux radioactive beams at isolde, Rev. Sci. Instrum. 90 (2019) 103313.

[11] W. Paul and H. Steinwedel, Ein neues massenspektrometer ohne magnetfeld, Z. Naturforschg. 8a (1953) 448.

[12] L.A. Pipes, Matrix solution of equations of the Mathieu-Hill type, J. Appl. Phys. 24 (1953) 902.

[13] F.M. Arscott, Periodic differential equations, The Macmillian company, U.S.A. (1964).

[14] A.A. Tovar and W. Casperson, Generalized Sylvester theorems for periodic applications in matrix optics, J. Opt. Sco. Amer. A 12 (1995) 578.

[15] N. Konenkov, M. Sudakov and D. Douglas, Matrix methods for the calculation of stability diagrams in quadrupole mass spectrometry, Amer. Soc. Mass Spectrom. 13 (2002) 597.

[16] T. Day Goodacre et al., Blurring the boundaries between ion sources: the application of the RILIS inside a FEBIAD type ion source at ISOLDE, Nucl. Instrum. Meth. B 378 (2016) 39.

[17] M. Haase, 5 kHz-100 mHz 4W amplifier (CERN BE-RF-IS 4222), CERN EDMS no. 1683282 (2016). 
[18] M. Paoluzzi and M. Haase, Amplifier assembly adjustment and testing procedure (CERN BE-RF-IS 4241), CERN EDMS no.2113766 (2016).

[19] M. Ady and R. Kersevan, Recent developments of Monte-Carlo codes Molflow+ and Synrad+, in the proceedings of the $10^{\text {th }}$ International Particle Accelerator Conference (IPAC2019), May 19-24, Melbourne, Australia (2019). 